\title{
Effect of the CXCR2 antagonist danirixin on symptoms and health status in COPD
}

\begin{abstract}
To the Editor:
Approximately one-third of patients with chronic obstructive pulmonary disease (COPD) have chronic mucus hypersecretion $(\mathrm{CMH})$ [1], often referred to as chronic bronchitis. Despite treatment with inhaled medications and other therapies, such as expectorants or methylxanthines, patients with $\mathrm{CMH}$ may continue to experience exacerbations, substantial symptom burden and poor health status [1]. CXCR2 antagonists are effective in multiple preclinical and human models of airway inflammation [2-5]. Danirixin is a competitive, reversible, oral CXCR2 antagonist that has been well tolerated in healthy subjects and in patients with influenza [6,7]. We report the results of a 52-week Phase 2 study conducted in Germany and the USA (GSK protocol 200163; ClinicalTrials.gov identifier NCT02130193) assessing the effects of danirixin when added to standard-of-care inhaled medications in participants with symptomatic COPD. Participants with a forced expiratory volume in $1 \mathrm{~s}$ ( $\mathrm{FEV} 1) \geqslant 50 \%$ of predicted normal and a history of two exacerbations in the prior 12 months, or one exacerbation and elevated plasma fibrinogen $\geqslant 3 \mathrm{mg} \cdot \mathrm{mL}^{-1}$, as well as a history of chronic cough and/or mucus hypersecretion on most days for at least the previous 3 months prior to screening, were eligible. Full inclusion and exclusion criteria are available online at clinicaltrials.gov. The study was approved by the Western Institutional Review Board (Puyallup, WA, USA) and the Ethikkommission I, Ärztekammer Schleswig-Holstein (Bad Segeberg, Germany). Written informed consent was obtained from all participants.
\end{abstract}

The primary end-points were respiratory symptoms at week 52 evaluated using the Evaluating Respiratory Symptoms in COPD (E-RS: COPD) instrument recorded in an electronic diary, and the incidence of healthcare resource utilisation (HCRU)-defined COPD exacerbations. The E-RS: COPD quantifies respiratory symptom severity and yields a total score, with higher scores indicating more severe symptoms. It is suggested that a reduction of two points or more is indicative of a clinically meaningful improvement in symptoms [8]. Key secondary end-points included change from baseline in both health status (COPD assessment test (CAT) and E-RS: COPD subscale scores (breathlessness, cough and sputum, and chest symptoms)) and safety.

All randomised participants $(n=93)$ were included in the intent-to-treat population for the primary analysis using a Bayesian repeated-measures mixed-effects model. The mean monthly E-RS: COPD score with corresponding 95\% credible intervals was calculated, along with the difference between treatment groups. The number of HCRU-defined exacerbations was analysed using a Bayesian Cox model. CAT scores were summarised for each treatment group at each visit, with a clinically meaningful improvement indicated by a decrease from baseline of $\geqslant 2$ points [9].

Following screening of 127 participants, 93 were randomised to receive either oral danirixin 75 mg twice daily $(n=45)$ or placebo $(n=48)$, in addition to COPD standard-of-care medications (figure 1a). Decreases in the E-RS: COPD total score were observed with danirixin within 2 months of study start and were maintained through 52 weeks (figure 1b). The posterior mean total score over months 3-12 was 11.16 in the danirixin group and 12.67 in the placebo group (treatment difference -1.52 (95\% CrI $-4.26-+1.33)$ ). In a post hoc analysis, the Bayesian probability that the true treatment difference over months $3-12$ was $<0$ was $85 \%$; the probability that the true treatment difference was $<-1$ was $65 \%$. The improvement in E-RS: COPD scores appeared to be driven by changes in the breathlessness and cough and sputum subscales, while no change was noted in the chest symptom subscale. At week 52, CAT scores had improved by a

@ERSpublications

Danirixin was associated with improved respiratory symptoms and health status in patients with mild-to-moderate COPD http://ow.ly/5m6P30luwfh

Cite this article as: Lazaar AL, Miller BE, Tabberer M, et al. Effect of the CXCR2 antagonist danirixin on symptoms and health status in COPD. Eur Respir J 2018; 52: 1801020 [https://doi.org/10.1183/ 13993003.01020-2018]. 
a)

\begin{tabular}{lcc}
\hline Baseline characteristic & $\begin{array}{c}\text { Danirixin } 75 \mathrm{mg} \text { twice daily } \\
(\mathrm{n}=45)\end{array}$ & $\begin{array}{c}\text { Placebo } \\
(\mathrm{n}=48)\end{array}$ \\
\hline Age years & $62.4 \pm 6.91$ & $58.8 \pm 7.32$ \\
Current smokers n (\%) & $34(76)$ & $34(71)$ \\
FEV $\%$ predicted & $67.2 \pm 15.1$ & $64.4 \pm 16.4$ \\
E-RS: COPD total score & $12.1 \pm 5.74$ & $12.1 \pm 5.79$ \\
CAT score & $19.2 \pm 6.02$ & $19.3 \pm 6.30$ \\
Concomitant medications at screening & & \\
$\quad$ ICS + LABA + LAMA & $33 \%$ & $50 \%$ \\
ICS + LABA & $16 \%$ & $17 \%$ \\
LABA + LAMA & $11 \%$ & $6 \%$ \\
LABA & $7 \%$ & $2 \%$ \\
LAMA & $11 \%$ & $6 \%$ \\
\hline
\end{tabular}

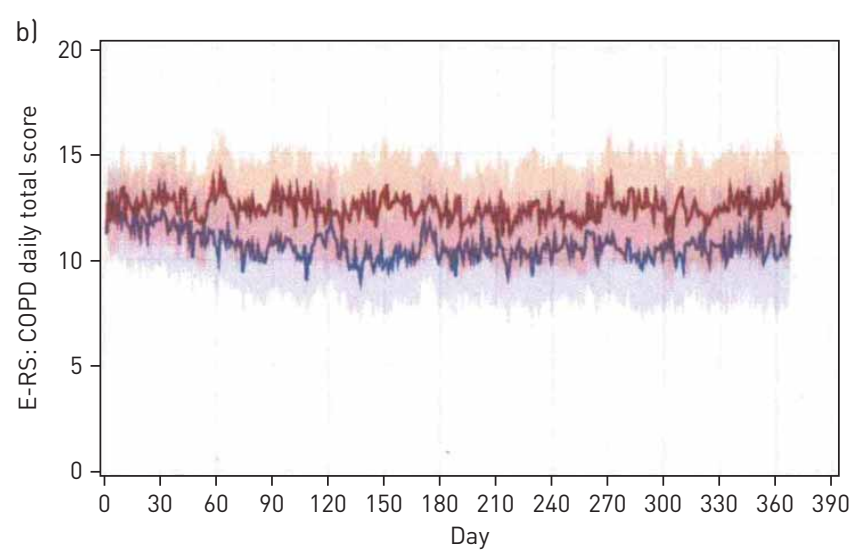

Treatment Danirixin 75 mg twice daily $\square$ Placebo

$\begin{array}{lllllllllllll}44 & 46 & 45 & 43 & 41 & 39 & 39 & 37 & 38 & 36 & 38 & 36 & 35\end{array}$

$\begin{array}{lllllllllllll}44 & 41 & 41 & 39 & 40 & 38 & 38 & 37 & 36 & 37 & 34 & 35 & 30\end{array}$ c)

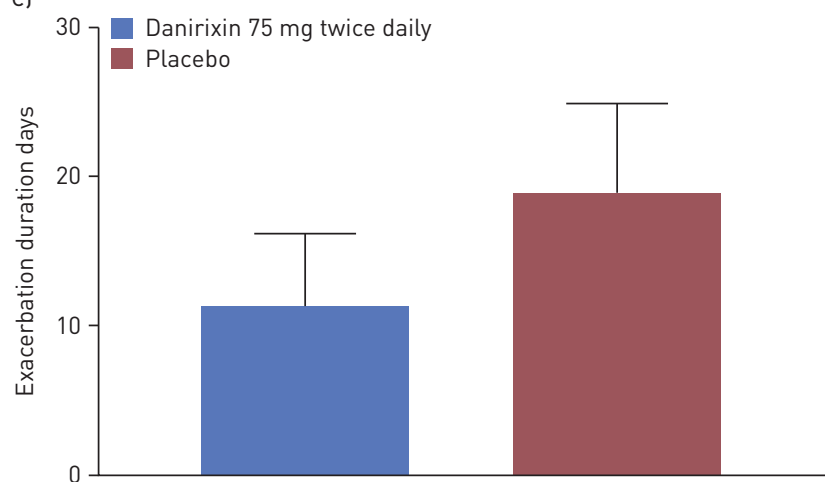

d)

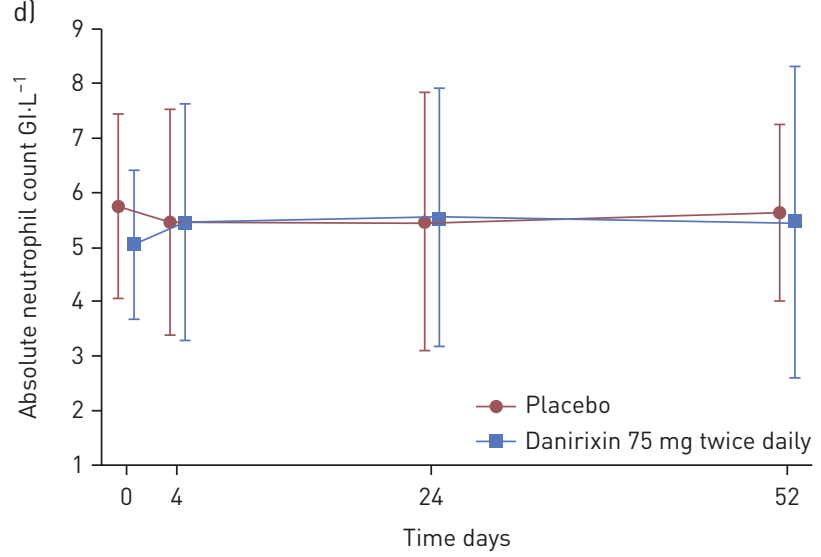

FIGURE 1 a) Patient demographics and baseline characteristics; b) daily Evaluating Respiratory Symptoms in Chronic Obstructive Pulmonary

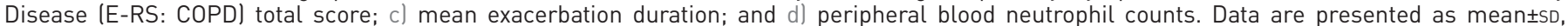

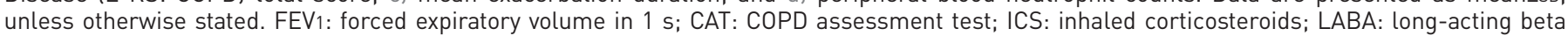
agonist; LAMA: long-acting muscarinic receptor antagonist.

mean of -2.1 points $(95 \% \mathrm{CI}-5.1-+1.0)$ in the danirixin group compared with a deterioration of +0.7 points $(95 \% \mathrm{CI}-1.2-+2.6)$ in the placebo group. In a post hoc analysis, the Bayesian probability that the true treatment difference at week 52 was $<-1$ was $90 \%$; the probability that the true treatment difference was $<-2$ was $71.7 \%$.

During the study, 21 (47\%) participants receiving danirixin and 23 (48\%) receiving placebo experienced at least one moderate or severe COPD exacerbation. There was no difference in the total number of HCRU-defined exacerbations in the danirixin and placebo groups (43 versus 39 exacerbations, respectively); however, the median reported exacerbation duration was 9 days (range 4-50) in the danirixin group and 17 days (range 4-65) in the placebo group (figure 1c).

Approximately $80 \%$ of participants in both treatment groups experienced at least one adverse event during the study, but few were drug-related (danirixin 4 (9\%) versus placebo 7 (15\%)). Diarrhoea, nausea and headache were the most common treatment-related adverse events in both groups, though reported at a lower incidence in the danirixin group. There was no difference in the number of participants with serious adverse events or withdrawals due to adverse events. A complete listing of adverse events and severe adverse events is available online at clinicaltrials.gov. No changes in blood neutrophil levels were observed with danirixin (figure 1d). The median serum C-reactive protein was $3.4 \mathrm{mg} \cdot \mathrm{L}^{-1}$ (interquartile range 1.8-6.4) at day 1 and $4.0 \mathrm{mg} \cdot \mathrm{L}^{-1}(1.6-6.7)$ at week 52 for the danirixin group; the corresponding values for placebo were $2.3 \mathrm{mg} \cdot \mathrm{L}^{-1}(1.4-5.9)$ and $3.5 \mathrm{mg} \cdot \mathrm{L}^{-1}(2.1-6.4)$, respectively.

This first-in-patient study investigated the safety and efficacy of danirixin in participants with COPD and $\mathrm{CMH}$ who were symptomatic, with a history of exacerbations despite receiving standard-of-care inhaled medications. Overall, participants who received danirixin demonstrated improvements in respiratory symptoms and health status compared with participants on placebo. We observed no difference in the 
number of HCRU-defined exacerbations experienced by participants in the danirixin and placebo groups, although the data raise the possibility that danirixin may reduce the duration of COPD exacerbations. This should be interpreted cautiously, as the methods for determining exacerbation duration were not standardised and probably varied from observer to observer. Respiratory symptom scores improved over time starting from about 2 months of treatment and there was an improvement in health status, demonstrated by a clinically meaningful decrease in the CAT score in participants treated with danirixin. Since no differences were observed in the number of HCRU-defined exacerbations experienced between the two treatment groups, this may indicate that the changes in health status scores are not driven by effects on acute exacerbations but by an overall reduction in the burden of lung inflammation. This will be explored in larger trials. The incidence of adverse events was similar in the danirixin- and placebo-treated groups. The absence of neutropenia seen with danirixin contrasts with other CXCR2 antagonists that have been tested in asthma, bronchiectasis and COPD [10-12] and may be due to the greater reversibility of its receptor binding compared with the other CXCR2 antagonists [5, 13-15].

The current trial has limitations, notably the small number of participants. A previous study suggested that CXCR2 antagonists may have greater efficacy in current rather than former smokers [11], but as most participants in the current study were smokers, it was not possible to analyse the effect of danirixin by smoking status. Finally, there were no E-RS: COPD scores collected prior to the start of treatment.

In conclusion, this study indicates positive trends in respiratory symptoms and health status in patients with mild-to-moderate symptomatic COPD at high risk for exacerbations and support the hypothesis that danirixin may be a useful adjunct treatment.

Aili L. Lazaar ${ }^{1}$, Bruce E. Miller ${ }^{1}$, Maggie Tabberer ${ }^{2}$, John Yonchuk $^{1}$, Nancy Leidy ${ }^{3}$, Claire Ambery ${ }^{2}$, Jackie Bloomer ${ }^{4}$, Henrik Watz ${ }^{5}$ and Ruth Tal-Singer ${ }^{1}$

${ }^{1}$ GSK, Collegeville, PA, USA. ${ }^{2}$ GSK, Stockley Park, UK. ${ }^{3}$ Evidera, Bethesda, MD, USA. ${ }^{4}$ GSK, Ware, UK. ${ }^{5}$ Pulmonary Research Institute Lungen Clinic Grosshansdorf, Airway Research Center North (ARCN), German Center for Lung Research (DZL), Grosshansdorf, Germany.

Correspondence: Aili L. Lazaar, 1250 S. Collegeville Road, Collegeville, PA 19426 USA. E-mail: aili.l.lazaar@gsk.com

This study is registered at ClinicalTrials.gov with identifier NCT02130193. Within 6 months of publication, anonymised individual participant data, the annotated case report form, protocol, reporting and analysis plan, data set specifications, raw dataset, analysis-ready dataset, and clinical study report will be available for research proposals approved by an independent review committee. A data access agreement will be required. Proposals should be submitted to www. clinicalstudydatarequest.com

Received: Jan 262018 | Accepted after revision: Aug 022018

Conflict of interest: A.L. Lazaar is an employee of GlaxoSmithKline and holds stock in the company. B.E. Miller is an employee and shareholder of GSK. M. Tabberer is an employee of and holds stock in GSK. J. Yonchuk is an employee and shareholder of GSK. N. Leidy is employed by Evidera, a healthcare research firm that provides consulting and other research services to pharmaceutical, device, government and non-government organisations. In this salaried position, N. Leidy works with a variety of companies and organisations, receiving no payment or honoraria directly from these organisations for services rendered, apart from honoraria received for her advisory role on two NIH-funded programmes: PATIENTS and PCAR. Evidera is the copyright owner of the EXACT and E-RS. C. Ambery is an employee and shareholder of GSK. J. Bloomer is an employee and shareholder of GSK. H. Watz is an employee of GSK. R. Tal-Singer is an employee and shareholder of GSK.

Support statement: This study was funded by GlaxoSmithKline. Funding information for this article has been deposited with the Crossref Funder Registry.

\section{References}

1 Ramos F, Krahnke JS, Kim V. Clinical issues of mucus accumulation in COPD. Int J Chron Obstruct Pulmon Dis 2014; 9: 139-150.

2 Holz O, Khalilieh S, Ludwig-Sengpiel A, et al. SCH527123, a novel CXCR2 antagonist, inhibits ozone-induced neutrophilia in healthy subjects. Eur Respir J 2010; 35: 564-570.

3 Lazaar AL, Sweeney LE, MacDonald AJ, et al. SB-656933, a novel CXCR2 selective antagonist, inhibits ex vivo neutrophil activation and ozone-induced airway inflammation in humans. Br J Clin Pharmacol 2011; 72: 282-293.

4 Busch-Petersen J, Carpenter DC, Burman M, et al. A reversible and selective antagonist of the CXC chemokine receptor 2. J Pharmacol Exp Ther 2017; 362: 338-346.

5 Pedersen F, Waschki B, Marwitz S, et al. Neutrophil extracellular trap formation is regulated by CXCR2 in COPD neutrophils. Eur Respir J 2018; 51: 1700970.

6 Miller BE, Mistry S, Smart K, et al. The pharmacokinetics and pharmacodynamics of danirixin (GSK1325756) - a selective CXCR2 antagonist - in healthy adult subjects. BMC Pharmacol Toxicol 2015; 16: 18-30.

7 GlaxoSmithKline. Safety, Tolerability and Clinical Effect in Adults with Influenza. Available from: https:// clinicaltrials.gov/ct2/show/results/NCT02469298. NLM Identifier NCT02469298. Date last accessed: December 27, 2017. 
8 Leidy NK, Murray LT, Monz BU, et al. Measuring respiratory symptoms of COPD: performance of the EXACTRespiratory Symptoms Tool (E-RS) in three clinical trials. Respir Res 2014; 15: 124.

9 Jones PW, Harding G, Wiklund I, et al. Tests of the responsiveness of the COPD assessment test following acute exacerbation and pulmonary rehabilitation. Chest 2012; 142: 134-140.

10 De Soyza A, Pavord I, Elborn JS, et al. A randomised, placebo-controlled study of the CXCR2 antagonist AZD5069 in bronchiectasis. Eur Respir J 2015; 46: 1021-1032.

11 Rennard SI, Dale DC, Donohue JF, et al. CXCR2 Antagonist MK-7123. A phase 2 proof-of-concept trial for chronic obstructive pulmonary disease. Am J Respir Crit Care Med 2015; 191: 1001-1011.

12 O’Byrne PM, Metev H, Puu M, et al. Efficacy and safety of a CXCR2 antagonist, AZD5069, in patients with uncontrolled persistent asthma: a randomised, double-blind, placebo-controlled trial. Lancet Respir Med 2016; 4: 797-806.

13 Hastrup N, Khalilieh S, Dale DC, et al. The effects of the CXCR2 antagonist, MK-7123, on bone marrow functions in healthy subjects. Cytokine 2015; 72: 197-203.

14 Chapman RW, Minnicozzi M, Celly CS, et al. A novel, orally active CXCR1/2 receptor antagonist, SCH527123, inhibits neutrophil recruitment, mucus production, and goblet cell hyperplasia in animal models of pulmonary inflammation. J Pharmacol Exp Ther 2007; 322: 486-493.

15 Nicholls DJ, Wiley K, Dainty I, et al. Pharmacological characterization of AZD5069, a slowly reversible CXC chemokine receptor 2 antagonist. J Pharmacol Exp Ther 2015; 353: 340-350. 